\title{
Retracted Tympanometry Result
}

National Cancer Institute

\section{Source}

National Cancer Institute. Retracted Tympanometry Result. NCI Thesaurus. Code C88508.

A tympanometry test result indicating the retraction of the tympanic membrane resulting from negative ear pressure. 